\title{
Nonradiative resonant excitation transfer from nanocrystal quantum dots to adjacent quantum channels
}

\author{
Siyuan $\mathrm{Lu}^{1}$ and Anupam Madhukar ${ }^{1,2,}$ \\ Nanostructure Materials and Devices Laboratory, \\ Department of Physics ${ }^{1}$ and \\ Mork Family Department of Chemical Engineering and Materials Science ${ }^{2}$ \\ University of Southern California, Los Angeles, CA 90089-0241
}

\section{Supporting Information}

\section{S1. Synthesis of Nanocrystal quantum dot/Quantum well hybrid structures}

We employed molecular beam epitaxy to grow a variety of InGaAs based quantum wells (QWs) and self-assembled quantum dot structures with the aim of tailoring their relative average energies and full widths at half maxima with respect to the photoluminescence (PL) peak and distribution of the NCQDs such as to allow for unambiguous measurements when exciting the composite structures. We found that utilizing $\left[(\mathrm{InAs})_{\mathrm{m}}(\mathrm{GaAs})_{\mathrm{n}}\right]$ short period superlattices allows a higher quality effective quantum well than the straight InGaAs alloy quantum wells for the higher $(\sim 30 \%)$ Indium content structures needed to tailor the quantum well band gap with respect to the HOMO-LUMO average gap 
of the PbS NCQDs. On semi-insulating GaAs substrates, first GaAs buffer was grown at standard conditions followed by a $\left[(\mathrm{InAs})_{1}(\mathrm{GaAs})_{2}\right] \mathrm{x} 8$ quantum well grown at $425^{\circ} \mathrm{C}$ substrate temperature under $1.5 \times 10^{-6}$ torr of arsenic flux equivalent pressure. Between each period of $(\operatorname{InAs})_{1}(\mathrm{GaAs})_{2}$, growth was interrupted for 10 seconds under arsenic flux. Finally a GaAs capping layer was grown at $400^{\circ} \mathrm{C}$ under arsenic pressure $1.5 \times 10^{-6}$ torr by migration enhanced epitaxy (MEE) method. During the QW growth, reflection high-energy electron diffraction pattern was monitored and remained streaky indicating that, as desired, the growth front remains atomically smooth in spite of the high (33\%) Indium composition of the QW.

The surface passivation of GaAs and NSQW bearing GaAs substrate surfaces was carried out by immersion in $5 \%\left(\mathrm{NH}_{4}\right)_{2} \mathrm{~S}$ aqueous solution at $50{ }^{\circ} \mathrm{C}$ for 10 minutes after an etch in $\mathrm{H}_{2} \mathrm{SO}_{4}: \mathrm{H}_{2} \mathrm{O}(3: 1)$. Care was taken to ensure the passivation procedures are exactly the same for GaAs and NSQW substrates. Atomic force microscopy (AFM) measurements (tapping mode, Digital Instrument MultiMode AFM) showed an rms surface roughness of $\sim 0.15 \mathrm{~nm}$ for passivated GaAs and passivated NSQW.

The $\mathrm{PbS}$ core nanocrystals (average core diameter $2.5 \mathrm{~nm}, \sim 950 \mathrm{~nm}$ emission peak) were purchased from Evident Technology (Troy, NY) and diluted with anhydrous toluene.

To create the NCQD/substrate hybrid structure samples, passivated GaAs and NSQW substrates were dipped into $2.5 \mathrm{mg} / \mathrm{ml} \mathrm{PbS}$ NCQDs toluene solution and pulled out at a speed of $\sim 5 \mu \mathrm{m} / \mathrm{sec}$ in $\mathrm{Ar}$ environment so that a sub one monolayer of closely packed NCQDs are deposited on the substrate as characterized by atomic force microscope (AFM). Figure S1 shows an illustrative AFM image of 
such a sample. After NCQD deposition, NCQD/substrate samples were transferred, without exposure to air, into a cryostat and pumped to $\sim 1 \times 10^{6}$ torr vacuum for photoluminescence measurement. Experiments showed that NCQDs on substrate samples can stayed in the cryostat for several weeks without significant change in the NCQD PL behavior.

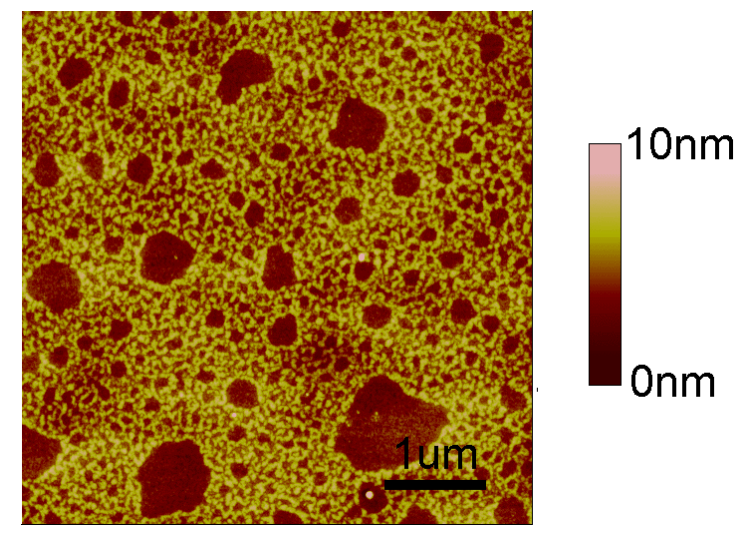

Figure S1. illustrative tapping mode AFM image of monolayer high submonolayer coverage of $\mathrm{PbS}$ NCQDs on GaAs surface.

\section{S2. Time-integrated and time-resolved PL measurement:}

For time-integrated PL measurements, a CW Ti:Sapphire tunable laser is used for excitation, photoluminescence is dispersed with a 1m double monochromator and detected using LN2 cooled Ge detector. Unless noted otherwise, the excitation power densities used in the PL/PLE measurement are $100 \mathrm{~W} / \mathrm{cm}^{2}$. At this low excitation power density, the exciton occupation in NCQDs is estimated to be less than 0.01 per NCQD. All PL/PLE data shown have their intensities normalized to the NCQD coverage of individual samples obtained from AFM measurements performed after the all PL/PLE measurements. 
For time-resolved PL (TRPL) measurement, an intracavity dumped mode-locked femto-second Ti:Sapphire laser were used for excitation. Photoluminescence is dispersed with $1 / 4 \mathrm{~m}$ subtractive monochromator and detected using a microchannel plate photomultiplier tube with S1 cathode. Time resolved PL spectrum were obtained using time-correlated single photon counting (TC-SPC) technique. While the minimum width of the instrument response function (IRF) of the TRPL setup is $\sim 40 \mathrm{ps}$, the IRF corresponding to the measurements shown in figure 4 and 6 of the manuscript is $\sim 2.5 \mathrm{~ns}$ (FWHM) due to the required $\mu$ s time range. The IRF is far shorter than the luminescence lifetime of the PbS NCQDs. Therefore the measured TRPL spectrum is used without deconvolution. 